Research Article

\title{
Solution of the $\kappa$-Deformed Dirac Equation with Vector and Scalar Interactions in the Context of Spin and Pseudospin Symmetries
}

\author{
Claudio F. Farias $\mathbb{D}$ and Edilberto O. Silva \\ Departamento de Física, Universidade Federal do Maranhão, 65085-580 São Luís, Maranhão, Brazil \\ Correspondence should be addressed to Edilberto O. Silva; edilbertoo@gmail.com
}

Received 27 September 2019; Accepted 9 December 2019; Published 1 February 2020

Academic Editor: Salvatore Mignemi

Copyright ( 92020 Claudio F. Farias and Edilberto O. Silva. This is an open access article distributed under the Creative Commons Attribution License, which permits unrestricted use, distribution, and reproduction in any medium, provided the original work is properly cited. The publication of this article was funded by SCOAP ${ }^{3}$.

\begin{abstract}
The deformed Dirac equation invariant under the $\kappa$-Poincaré-Hopf quantum algebra in the context of minimal and scalar couplings under spin and pseudospin symmetry limits is considered. The $\kappa$-deformed Pauli-Dirac Hamiltonian allows us to study effects of quantum deformation in a class of physical systems, such as a Zeeman-like effect, Aharonov-Bohm effect, and an anomalous-like contribution to the electron magnetic moment, between others. In our analysis, we consider the motion of an electron in a uniform magnetic field and interacting with (i) a planar harmonic oscillator and (ii) a linear potential. We verify that the particular choice of a linear potential induces a Coulomb-type term in the equation of motion. Expressions for the energy eigenvalues and wave functions are determined taking into account both symmetry limits. We verify that the energies and wave functions of the particle are modified by the deformation parameter as well as by the element of spin.
\end{abstract}

\section{Introduction}

Quantum deformations based on the $\kappa$-Poincaré-Hopf algebra constitute an important branch of research that enables us to address problems in condensed matter and high energy physics through field equations. A pioneer work presenting these equations can be viewed in Ref. [1] (see also Refs. [2-6]), where a new real quantum Poincaré algebra with a standard real structure is obtained by contraction of $U_{q}(O(3,2))$. The resulting algebra of this contraction is a standard real Hopf algebra that depends on a dimension-full parameter $\kappa$ instead of the real deformation parameter $q$. This algebra is defined by the following commutation relations:

$$
\begin{aligned}
{\left[p_{v}, p_{\mu}\right] } & =0, \quad \mu, v=0,1,2,3, \\
{\left[\tilde{M}_{i}, p_{\mu}\right] } & =\left(1-\delta_{0 \mu}\right) i \varepsilon_{i j k} p_{k}, \quad i, j=1,2,3, \\
{\left[L_{i}, p_{\mu}\right] } & =i\left[p_{i}\right]^{\delta_{0 \mu}}\left[\delta_{i j} \varepsilon^{-1} \sinh \left(\varepsilon p_{0}\right)\right]^{1-\delta_{0 \mu},} \\
{\left[\tilde{M}_{i}, \tilde{M}_{j}\right] } & =i \varepsilon_{i j k} \tilde{M}_{k}, \\
{\left[\tilde{M}_{i}, L_{j}\right] } & =i \varepsilon_{i j k} L_{k}, \\
{\left[L_{i}, L_{j}\right] } & =-i \varepsilon_{i j k}\left[\tilde{M}_{k} \cosh \left(\varepsilon p_{0}\right)-\frac{\varepsilon^{2}}{4} p_{k} p_{l} \tilde{M}_{l}\right],
\end{aligned}
$$

where $\varepsilon$ is defined by 


$$
\varepsilon=\kappa^{-1}=\lim _{R \rightarrow \infty}(R \ln q)
$$

with $R$ is being the de Sitter curvature and $p_{\mu}=\left(p_{0}, \mathbf{p}\right)$ are the $\kappa$-deformed generators for energy and momenta. In the above commutation relations, $\tilde{M}_{i}$ and $L_{i}$ represent the spatial rotations and deformed boost generators, respectively. The coalgebra and antipode for the $\kappa$-deformed Poincaré algebra were established in Ref. [7] and have the following representation:

$$
\begin{aligned}
\Delta \tilde{M}_{i}= & \tilde{M}_{i} \otimes I+I \otimes \tilde{M}_{i}, \\
\Delta p_{0}= & p_{0} \otimes I+I \otimes p_{0}, \\
\Delta p_{i}= & p_{i} \otimes e^{(1 / 2) \varepsilon p_{0}}+e^{-(1 / 2) \varepsilon p_{0} \otimes p_{i},} \\
\Delta L_{i}= & L_{i} \otimes e^{(1 / 2) \varepsilon p_{0}}+e^{-(1 / 2) \varepsilon p_{0} \otimes L_{i}} \\
& +\frac{1}{2} \varepsilon \varepsilon_{i j k}\left[p_{j} \otimes \tilde{M}_{k} e^{(1 / 2) \varepsilon p_{0}}+e_{j}^{-(1 / 2) \varepsilon p_{0}} \tilde{M} \otimes p_{k}\right], \\
S\left(p_{\mu}\right)= & -p_{\mu}, \\
S\left(\tilde{M}_{i}\right)= & -\tilde{M}_{i}, \\
S\left(L_{i}\right)= & -L_{i}+\frac{3 \varepsilon i}{2} p_{i},
\end{aligned}
$$

where $I$ is the identity operator. The deformed Casimir operators for the $\kappa$-Poincaré algebra have the following representation:

$$
\begin{aligned}
C_{1} & =\left[\frac{2}{\varepsilon} \sinh \left(\varepsilon p_{0}\right)\right]^{2}-p_{i} p_{i}, \\
C_{2} & =\left[\cosh \left(\varepsilon p_{0}\right)-\frac{\varepsilon^{2}}{4} p_{i} p_{i}\right] W_{0}^{2}-W_{i} W_{i},
\end{aligned}
$$

where

$$
\begin{aligned}
& W_{0}=p_{i} \tilde{M}_{i} \\
& W_{i}=\frac{1}{\varepsilon} \sinh \left(\varepsilon p_{0}\right) \tilde{M}_{i}+\varepsilon_{i j k} p_{j} p_{k} .
\end{aligned}
$$

With these definitions in hand, we write

$$
\begin{aligned}
\mathscr{P}_{\mu} & =p_{\mu} \\
\mathscr{M}_{i} & =\tilde{M}_{i}+m_{i}, \\
\mathscr{L}_{i} & =L_{i}+e^{-(1 / 2) \varepsilon p_{0}} l_{i}-\frac{1}{2} \varepsilon \varepsilon_{i j k} m_{j} p_{k}, \\
m_{i} & =\frac{1}{4} i \varepsilon_{i j k} \gamma_{j} \gamma_{k}, \\
l_{i} & =-\frac{1}{2} i \gamma_{0} \gamma_{i} .
\end{aligned}
$$

The $\kappa$-deformed Dirac operator derived from the above algebra is found to be

$$
\mathscr{D}=-e^{-(1 / 2) \varepsilon p_{0}} \gamma_{i} p_{i}+\frac{1}{\varepsilon} \gamma_{0} \sinh \left(\varepsilon p_{0}\right)-\frac{1}{2} \varepsilon \gamma_{0} p_{i} p_{i}
$$

and satisfies the following relations:

$$
\left[\mathscr{D}, \mathscr{P}_{\mu}\right]=\left[\mathscr{D}, \mathscr{M}_{i}\right]=\left[\mathscr{D}, \mathscr{L}_{i}\right]=0 .
$$

The square of operator (7) can be written in terms of the invariants $C_{1}$ and $C_{2}$ as

$$
\mathscr{D}^{2}=C_{1}\left(1+\frac{1}{4} \varepsilon^{2} C_{1}\right),
$$

or

$$
\mathscr{D}=\sqrt{C_{1}\left(1+\frac{1}{4} \varepsilon^{2} C_{1}\right)}=-\frac{4}{3} C_{2} .
$$

Since $C_{1}=M^{2}$, we can write

$$
\mathscr{D}=M \sqrt{\left(1+\frac{1}{4} \varepsilon^{2} M^{2}\right)} .
$$

In this way, the Dirac equation can be written as

$$
\mathscr{D} \psi-M \sqrt{1+\frac{1}{4} \varepsilon^{2} M^{2}} \psi=0 .
$$

For more details on the derivation of equation (12), see [3], for example. The algebraic structure of the $\kappa$-deformed Poincaré algebra has been widely investigated intensively and has become a theoretical field of increasing interest $[5,6,8-25]$. Through the field equations from the $\kappa$-Poincaré algebra ( $\kappa$-Dirac equation $[3,4,26])$, we can study the physical implications of the quantum deformation parameter $\kappa$ in relativistic and nonrelativistic quantum systems. In this context, we highlight the study of relativistic Landau levels [22], the Aharonov-Bohm effect taking into account spin effects [21], the Dirac oscillator [27, 28], and the integer quantum Hall effect [29].

When we want to study the relativistic quantum dynamics of particles with spin, it is desirable to consider the presence of external fields, which include the vector and scalar fields. The inclusion of vector and scalar potentials in the Dirac equation reveals interesting properties of symmetries in nuclear theory. The first contributions in this subject revealed the existence of $\mathrm{SU}(2)$ symmetries, which are known in the literature as pseudospin and spin symmetries [30, 31]. Some investigations have been made in this scenario in order to give a meaning to these symmetries. However, it was only in a work by Ginocchio that pseudospin symmetry was revealed. He verified that pseudospin symmetry in nuclei could arise from nucleons moving in a relativistic mean field, which has an attractive scalar and repulsive vector potential nearly equal in magnitude [32] (for a more detailed description, see Ref. [33]). Spin and pseudospin symmetries in the Dirac equation have been studied under different aspects in 
recent years (see Refs. [34, 35]). Some studies have been developed taking into account the exact spin and pseudospin symmetry limits to study the relativistic dynamics of physical systems interacting with a class of potentials [36-43].

The present work is proposed to investigate the $\kappa$ -deformed Dirac equation derived in Ref. [4] in the context of minimal and scalar couplings under spin and pseudospin symmetry limits. The structure of the paper is as follows: In Section 2 , we present the $\kappa$-deformed Dirac equation with couplings from which we derive the $\kappa$-deformed PauliDirac equation, by using the usual procedure that consists of squaring the $\kappa$-deformed Dirac equation. In Section 3, we consider the equation of Pauli and establish the spin and pseudospin symmetry limits. As an application, we consider the particle interacting with a uniform magnetic field in the $z$-direction in two different physical situations: (i) particle interacting with a harmonic oscillator and (ii) particle interacting with a linear potential. We obtain expressions for the energy eigenvalues and wave functions in both limits. In Section 4, we present our comments and conclusions.

\section{The $\kappa$-Deformed Dirac Equation with Couplings}

In this section, we introduce the minimal and scalar substitutions into $\kappa$-deformed Dirac equation (12). Before doing this, let us write it in a more convenient way to be treated. This can be accomplished by multiplying the left of equation (12) by $e^{-(1 / 2) \varepsilon p_{0}}$ and retaining terms up to $\mathcal{O}(\varepsilon)$. The resulting equation is found to be $[3,4]$

$$
\left\{\left(\gamma_{0} p_{0}-\gamma_{i} p_{i}\right)+\frac{1}{2} \varepsilon\left[\gamma_{0}\left(p_{0}^{2}-p_{i} p_{i}\right)-M p_{0}\right]\right\} \psi=M \psi
$$

Deformed Dirac equation (13) is invariant under the $\kappa$ -deformed Poincaré quantum algebra. Manipulating this equation and using the properties of the $\gamma$ Dirac matrices and then rearranging the terms, it is possible to write it in the Hamiltonian form, $(H-E) \psi=0$, where $H$ is the $\kappa$ -deformed Dirac Hamiltonian and $E$ are the energy eigenvalues. After identifying $p_{0}=H_{0}=E$, with $H_{0}=\gamma_{0} \gamma_{i} p_{i}+\gamma_{0}$ $M$, we can iterate equation (13). This development yields to

$$
\begin{aligned}
E \psi= & \gamma_{0} \gamma_{i} p_{i}+\gamma_{0} M-\frac{\varepsilon}{2}\left[\left(\gamma_{0} \gamma_{i} p_{i}+\gamma_{0} M\right)^{2}-p_{i} p_{i}\right] \\
& +\frac{\varepsilon}{2}\left[\gamma_{0} M\left(\gamma_{0} \gamma_{i} p_{i}+\gamma_{0} M\right)\right] \psi,
\end{aligned}
$$

where we have discarded the terms of order equal and superior to $\varepsilon^{2}$ on the iterative replacements of the energy $p_{0}$. The interactions can be introduced through the following prescriptions [44]:

$$
\begin{gathered}
p_{i} \longrightarrow p_{i}-e A_{i}, \\
E \longrightarrow E-V(r), \\
M \longrightarrow M+S(r),
\end{gathered}
$$

where $V(r)$ is the electric potential, $A_{i}$ is the vector potential, and $S(r)$ is the scalar potential. It is known from relativistic quantum mechanics that for vector coupling the potential couples to the charge (which is different for particles and antiparticles) while for scalar coupling the potential couples to the mass (which is equal for both particles and antiparticles).

As mentioned in Ref. [4], the couplings ((15), (16), and (17)) are quite satisfactory from the point of view of the $\kappa$-Poincaré-Hopf algebra. Indeed, there are no operator ordering problems after the gauging that would require symmetrization in equation (13). Using these couplings, we obtain the following expression:

$$
\begin{aligned}
& {\left[\gamma_{0} \gamma_{i}\left(p_{i}-e A_{i}\right)+\gamma_{0}(M+S(r))\right] \psi} \\
& \quad-\frac{1}{2} \varepsilon\left[\left[\gamma_{0} \gamma_{i}\left(p_{i}-e A_{i}\right)+\gamma_{0}(M+S(r))\right]^{2}\right] \psi \\
& \quad+\frac{1}{2} \varepsilon(M+S(r))\left[\gamma_{i}\left(p_{i}-e A_{i}\right)+(M+S(r))\right] \psi \\
& \quad+\frac{1}{2} \varepsilon\left(p_{i}-e A_{i}\right)^{2} \psi=(E-V(r)) \psi
\end{aligned}
$$

Since we are interested in planar dynamics, i.e., when both $p_{z}$ and $z$ as well as the third directions of the fields involved are zero, we choose the following representation for the gamma matrices in terms of the Pauli's matrices [45]:

$$
\begin{aligned}
& \gamma_{0}=\sigma_{3}, \\
& \alpha_{1}=\gamma_{0} \gamma_{1}=\sigma_{1}, \\
& \alpha_{2}=\gamma_{0} \gamma_{2}=s \sigma_{2},
\end{aligned}
$$

where parameter $s$, which has a value of twice the spin value, can be introduced to characterizing the two spin states, with $s=+1$ for spin $u p$ and $s=-1$ for spin down. The matrices $\sigma_{i}$ $(i=1,2,3)$ satisfy the properties

$$
\begin{aligned}
\sigma_{i}^{2} & =I, \\
{\left[\sigma_{i}, \sigma_{j}\right] } & =2 i \varepsilon_{i j k} \sigma_{k}, \\
\left\{\sigma_{i}, \sigma_{j}\right\} & =2 \delta_{i j}, \\
\sigma_{i} \sigma_{j} & =i \varepsilon_{i j k} \sigma_{k}+\delta_{i j}, \\
\sigma_{i} \sigma_{k} \sigma_{l} & =i \varepsilon_{i k l} I+\delta_{i k} \sigma_{l}+\delta_{k l} \sigma_{i} .
\end{aligned}
$$

Using the representation and the properties of the Pauli's matrices above, the $\kappa$-deformed Dirac equation including the interactions can be written as

$$
\begin{aligned}
{\left[\alpha \cdot(\mathbf{p}-e \mathbf{A})+\gamma_{0}(M+S(r))\right] \psi-[E-V(r)] \psi } \\
\quad+\frac{\varepsilon}{2}\left[e s(\sigma \cdot \mathbf{B}) \psi+\gamma_{0}((\alpha \cdot \mathbf{p}) S(r))\right] \psi \\
+\frac{\varepsilon}{2}\left[M \gamma_{0}(\alpha \cdot \mathbf{p})+\gamma_{0} S(r)(\alpha \cdot \mathbf{p}) \psi-\gamma_{0} e(\alpha \cdot \mathbf{A}) M \psi\right] \\
\quad-\frac{\varepsilon}{2}\left[\gamma_{0} e(\alpha \cdot \mathbf{A}) S(r) \psi\right]=0,
\end{aligned}
$$

where the magnetic field $B$ emerges when we use the relation 


$$
i \varepsilon_{i j k} \sigma_{k}\left(p_{i}-e A_{i}\right)\left(p_{j}-e A_{j}\right) \psi=-s \sigma_{k} B_{k} \psi
$$

Let us now determine the Dirac equation in its quadratic form. This can be accomplished by applying the matrix operator $[46,47]$

$$
\begin{aligned}
& \alpha \cdot(\mathbf{p}-e \mathbf{A})+\gamma_{0}(M+S(r))+E-V(r) \\
& \quad+\frac{\varepsilon}{2}\left[\gamma_{0} S(r)(\alpha \cdot \mathbf{p})+e s(\sigma \cdot \mathbf{B})+\gamma_{0}((\alpha \cdot \mathbf{p}) S(r))\right] \\
& \quad+\frac{\varepsilon}{2}\left[M \gamma_{0}(\alpha \cdot \mathbf{p})-\gamma_{0} e(\alpha \cdot \mathbf{A}) M-\gamma_{0} e(\alpha \cdot \mathbf{A}) S(r)\right]
\end{aligned}
$$

in equation (21). In this procedure, we follow a similar algebra and the same mathematical properties of the parameters and variables involved on the deduction of equation (21). The result is the $\kappa$-deformed Dirac-Pauli equation

$$
\begin{aligned}
(\mathbf{p} & -e \mathbf{A})^{2} \psi+\alpha \cdot[\mathbf{p} V(r)] \psi-\gamma_{0} \alpha \cdot[\mathbf{p} S(r)] \psi \\
& +[M+S(r)]^{2} \psi-[E-V(r)]^{2} \psi-e s \sigma_{z} B \psi \\
& -\frac{\varepsilon}{2}\left\{\gamma_{0}\left[\mathbf{p}^{2} S(r)\right]+\gamma_{0}[(\alpha \cdot \mathbf{p}) S(r)][(\alpha \cdot \mathbf{p})]\right\} \psi \\
& +\frac{\varepsilon}{2}\left\{2 \mathrm{is} \gamma_{0}[\sigma \cdot[(\mathbf{p} S(r)) \times \mathbf{p}]-2 i s e \sigma \cdot(\mathbf{p} S(r)) \times \mathbf{A}]\right\} \psi \\
& +\frac{\varepsilon}{2}\left\{e \gamma_{0}[(\alpha \cdot \mathbf{p}) S(r)][(\alpha \cdot \mathbf{A})]-S(r)[(\alpha \cdot \mathbf{p}) S(r)]\right\} \psi \\
& +\frac{\varepsilon}{2}\{2 M e s B+2 S(r) e s B-M[(\alpha \cdot \mathbf{p}) S(r)]\} \psi \\
& +\frac{\varepsilon}{2}\left\{M \gamma_{0}[(\alpha \cdot \mathbf{p}) V(r)]+\gamma_{0} S(r)[(\alpha \cdot \mathbf{p}) V(r)]\right\} \psi=0 .
\end{aligned}
$$

It can be easily verified in equation (24) that after considering $\varepsilon=0$, the resulting equation is the one well known in the literature (see, for example, Ref. [48]). In order to apply equation (24) to a given physical system, we need to choose a representation for the vector potential $\mathbf{A}$ and the scalar potentials $S(r)$ and $V(r)$. For some particular choices of these quantities, we can study the physical implications of quantum deformation on the properties of various physical systems of interest.

For the field configuration, we consider a constant magnetic field along the $z$-direction (in cylindrical coordinates), $\mathbf{B}=B \widehat{\mathbf{z}}$, which is obtained from the vector potential (in the Landau gauge) [49],

$$
\mathbf{A}=\frac{B r}{2} \widehat{\varphi}
$$

In this configuration, equation (24) reads

$$
X+\frac{\varepsilon}{2} Y=0
$$

with

$$
\begin{aligned}
X= & -\frac{\partial^{2} \psi}{\partial r^{2}}-\frac{1}{r} \frac{\partial \psi}{\partial r}-\frac{1}{r^{2}} \frac{\partial^{2} \psi}{\partial \varphi^{2}}+i e B \frac{\partial \psi}{\partial \varphi}+\frac{1}{4} e^{2} B^{2} r^{2} \psi \\
& +[M+S(r)]^{2} \psi-[E-V(r)]^{2} \psi-e s \sigma_{z} B \psi \\
& +i\left[\frac{\partial S(r)}{\partial r}\right] \gamma_{0} \alpha_{r} \psi-i\left[\frac{\partial V(r)}{\partial r}\right] \alpha_{r} \psi \\
Y= & \gamma_{0}\left[\frac{\partial^{2} S(r)}{\partial r^{2}}+\frac{1}{r} \frac{\partial S(r)}{\partial r}\right] \psi-\gamma_{0}\left[\frac{\partial S(r)}{\partial r}\right] \frac{\partial \psi}{\partial r} \\
& -i s\left[\frac{1}{r} \frac{\partial S(r)}{\partial r}\right] \frac{\partial \psi}{\partial \varphi}-2 \mathrm{is}\left[\frac{1}{r} \frac{\partial S(r)}{\partial r}\right] \frac{\partial \psi}{\partial \varphi} \\
& -i S(r) \gamma_{r}\left[\frac{\partial V(r)}{\partial r}\right] \psi+\mathrm{iS}(r) \alpha_{r}\left[\frac{\partial S(r)}{\partial r}\right] \psi \\
& +e s\left[\frac{\partial S(r)}{\partial r}\right] \frac{B r}{2} \psi-2 e s\left[\frac{\partial S(r)}{\partial r}\right] \frac{B r}{2} \psi \\
& +2 M e s B \psi+2 S(r) e s B \psi, \\
& -i M \gamma_{r}\left[\frac{\partial V(r)}{\partial r}\right] \psi+i M \alpha_{r}\left[\frac{\partial S(r)}{\partial r}\right] \psi \\
& 2 M
\end{aligned}
$$

where the matrices (19) are now given in cylindrical coordinates, $\gamma_{r}=i \sigma_{\varphi}, \gamma_{\varphi}=-$ is $\sigma_{r}$, with [48]

$$
\begin{aligned}
& \alpha_{r}=\gamma_{0} \gamma_{r}=\left(\begin{array}{cc}
0 & e^{-i s \varphi} \\
e^{i s \varphi} & 0
\end{array}\right), \\
& \alpha_{\varphi}=\gamma_{0} \gamma_{\varphi}=\left(\begin{array}{cc}
0 & -i e^{-i s \varphi} \\
i e^{i s \varphi} & 0
\end{array}\right), \\
& \gamma_{0}=\sigma_{z}=\left(\begin{array}{cc}
1 & 0 \\
0 & -1
\end{array}\right) .
\end{aligned}
$$

We will attribute expressions to functions $V(r)$ and $S(r)$ in equation (26) in the next section, when we treat the analysis of spin and pseudospin symmetries. We will argue after that only some particular choices for these functions will lead to a differential equation that admits an exact solution.

\section{Symmetry Limits}

To implement the spin and pseudospin symmetry limits, we make in equation (26) the requirement that $S(r)= \pm V(r)$, where the plus (minus) signal refers to spin (pseudospin) symmetry, respectively [32]. Next, by using $\psi=\left(\psi_{+}, \psi_{-}\right)^{T}$, the first and second lines in equation (26) can be written in a simple form, which allows us to solve them separately. Furthermore, as mentioned above, we need to choose a representation for the radial function $V(r)$. We give a representation in terms of cylindrically symmetric scalar potentials which lead to results well-known in the literature. 
3.1. Particle Interacting with a Harmonic Oscillator. It is well known that the harmonic oscillator can be used to describe a large number of physical systems. The interest in this issue appears in physical systems at an atomic scale and extends to high energy physics. It appears, for instance, as the interaction between atoms in the elastic crystal [50-52], as the effective potential acting on electrons moving in a uniform magnetic field (Landau levels) $[49,53-57]$ and in the quantization of the electromagnetic field [58]. The potential of the harmonic oscillator is included into equation (26) by making $V(r)= \pm S(r)=a r^{2}$, where $a$ is a constant. One can check that the spin symmetry is sufficient to decouple the radial equation that comes from the upper spinor component while the pseudospin symmetry decouples the radial equation that comes from the lower component of the spinor. Thus, by adopting solutions of the form

$$
\psi_{ \pm}=\left(\begin{array}{c}
\sum_{m} f_{+}(r) e^{i m \varphi} \\
i \sum_{m} f_{-}(r) e^{i(m+s) \varphi}
\end{array}\right),
$$

we arrive at radial equations

$$
\begin{aligned}
\frac{d^{2} f_{ \pm}(r)}{d r^{2}} & +\left(\frac{1}{r}+\varepsilon a r\right) \frac{d f_{ \pm}(r)}{d r}-\frac{\left(m^{ \pm}\right)^{2}}{r^{2}} f_{ \pm}(r) \\
& -\left(\Omega^{ \pm}\right)^{2} r^{2} f_{+}(r)+k^{ \pm} f_{+}(r)=0,
\end{aligned}
$$

where $\quad k^{+}=E^{2}-M^{2}+2(m+s) \omega-\varepsilon(2 a+3 s m a+2 M \omega s)$, $k^{-}=E^{2}-M^{2}+2 \omega(m+s)+2 \omega s-\varepsilon[2 a-3 s a(m+s)+2 M \omega s]$, $\left[\Omega^{+}\right]^{2}=\omega^{2}+2(M+E) a-\varepsilon \omega s a, \quad\left[\Omega^{-}\right]^{2}=\omega^{2}+2(E-M) a-\varepsilon$ $\omega s a, \omega=e B / 2, m^{+}=m$, and $m^{-}=m+s$. It is convenient to write equation (30) in a known canonical form. This can be accomplished using $f_{ \pm}(r)$ as

$$
f_{ \pm}(\rho)=e^{-(1 / 2)\left(\kappa^{ \pm}+1\right) \rho} \rho^{(1 / 2)\left|m^{ \pm}\right|} F_{ \pm}(\rho), \quad \rho=\Omega^{ \pm} r^{2},
$$

where $\kappa^{ \pm}=\varepsilon a / 2 \Omega^{ \pm}$, which leads to the equation

$$
\begin{aligned}
\rho \frac{d^{2} F_{ \pm}}{d \rho^{2}} & +\left(1+\left|m^{ \pm}\right|-\rho\right) \frac{d F_{ \pm}}{d \rho} \\
& -\left[\frac{1}{2}\left(1+\left|m^{ \pm}\right|+\kappa^{ \pm}\right)-\frac{k^{ \pm}}{4 \Omega^{ \pm}}\right] F_{ \pm}=0 .
\end{aligned}
$$

Equation (32) is of the confluent hypergeometric equation type, and its solution is given in terms of the Kummer functions. In this manner, the general solution for equation (32) is given by [59]

$$
\begin{aligned}
f_{ \pm}(\rho)= & c_{1} e^{-(1 / 2)\left(1+\kappa^{ \pm}\right) \rho} \rho^{(1 / 2)\left|m^{ \pm}\right|} \\
& \times M\left(\frac{1}{2}\left(1+\left|m^{ \pm}\right|+\kappa^{ \pm}\right)-\frac{k^{ \pm}}{4 \Omega^{ \pm}}, 1+\left|m^{ \pm}\right|, \rho\right) \\
& +c_{2} e^{-(1 / 2)\left(1+\kappa^{ \pm}\right) \rho} \rho^{-(1 / 2)\left|m^{ \pm}\right|} \\
& \times M\left(\frac{1}{2}\left(1-\left|m^{ \pm}\right|+\kappa^{ \pm}\right)-\frac{k^{ \pm}}{4 \Omega^{ \pm}}, 1-\left|m^{ \pm}\right|, \rho\right),
\end{aligned}
$$

where $M$ are the Kummer functions. In particular, when $\left(1+\left|m^{ \pm}\right|+\kappa^{ \pm}\right) / 2-k^{ \pm} / 4 \Omega^{ \pm}=-n$, with $n=0,1,2, \cdots$, the function $M$ becomes a polynomial in $\rho$ of degree not exceeding $n$. From this condition, we extract the energies for the spin and pseudospin symmetry limits, given, respectively, by

$$
\begin{aligned}
E^{2}-M^{2}= & 2 \sqrt{\omega^{2}+2(M+E) a-\varepsilon \omega s a}(2 n+|m|+1) \\
& +\varepsilon(3 a+3 s m a+2 \omega M s)-2 \omega(m+s), \\
E^{2}-M^{2}= & 2 \sqrt{\omega^{2}+2(E-M) a+\varepsilon \omega s a}(2 n+1+|m+s|) \\
& -2 \omega(m+s)-2 \omega s-\varepsilon[a-3 s a(m+s)+2 M \omega s] .
\end{aligned}
$$

Equations (34) and (35) are, respectively, the particle and antiparticle energies in the context of quantum deformation, and they can be read as a relativistic generalization of the Landau levels. It must be emphasized that, since $\varepsilon$ and $a$ are positive, the quantum deformation affects the separation of the energy levels of the system. This feature, however, should not depend on the value of the spin projection parameter $s$. Figure 1 shows energy profile (34) as a function of the frequency $\omega$ for some values of the quantum number $m$. In Figure 1(a), we plotted for $s=1$ and in Figure 1(b) for $s=-1$. In this analysis as well as for the others, we use $\varepsilon=10^{-7}[18,29]$. For this value, the effects of quantum deformation become more evident. We clearly see that both particle and antiparticle belong to the same energy spectrum. However, in Figure 1(b), we find that the antiparticle energy is not defined in the frequency ranges $0<\omega<1.37$ (for $m=0$ ) and $0<\omega<2$ (for $m=-1$ ). These same characteristics are also present in the energy profile of equation (35) as shown in Figure 2. However, in Figure 2(b), the energies are not defined in the frequency ranges $0<\omega<3.35$ (for $m=-1$ ), $0<\omega<3.1$ (for $m=-1$ ), and $0<\omega<2.65$ (for $m$ $=1$ ). The appearance of such regions characterizing the absence of energy eigenvalues is due to the quantum deformation effects present in the model. On the other hand, when we compare the energy (34) (spin symmetry case) with the energy (7) of Ref. [28], we observe the absence of the interaction term between the Dirac oscillator and the angular momentum. Here, the frequency $\omega$ comes from the gauge field (25), which couples with the spin of the particle. Although these spectra are similar, physically, they are different. In fact, when $\varepsilon=0$, we obtain 


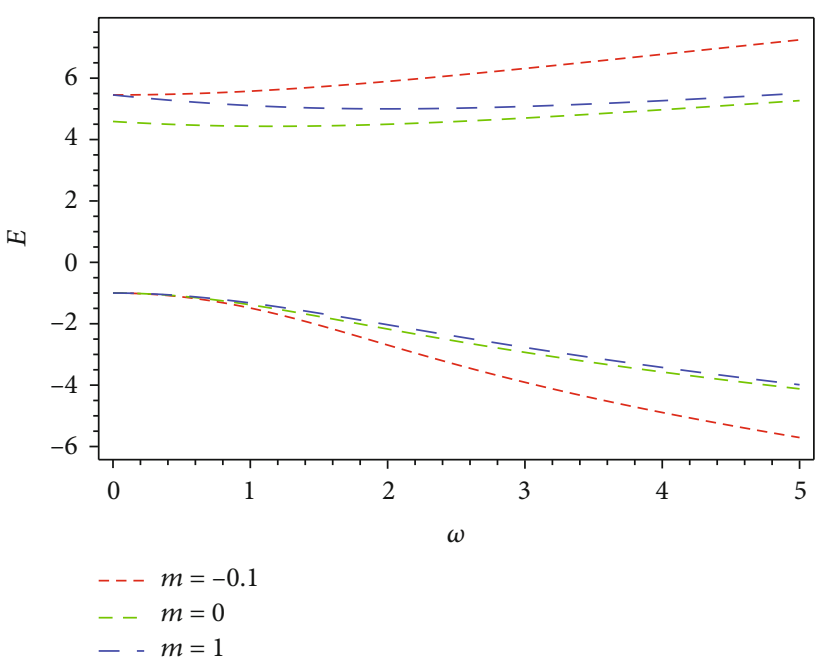

(a)

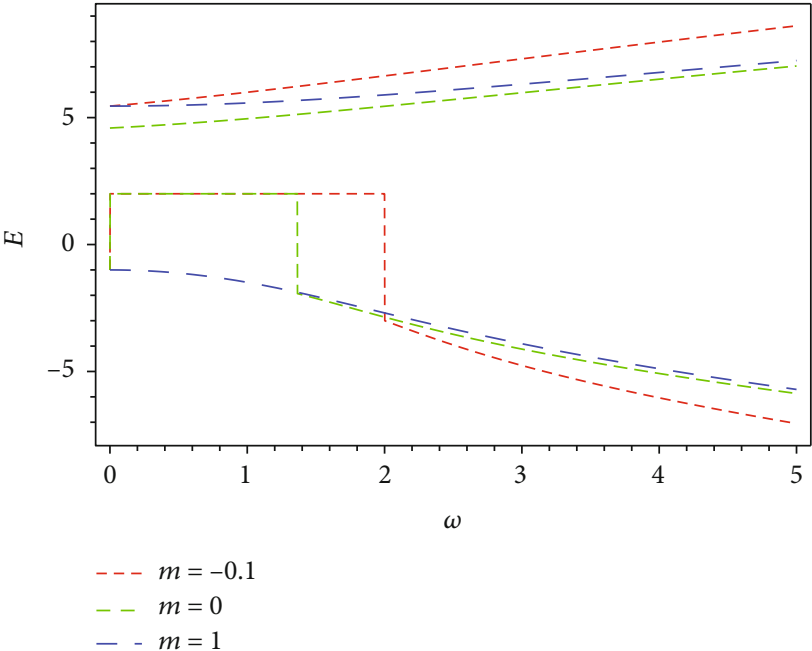

(b)

FIgURE 1: Illustration of the energy eigenvalues in the spin symmetry limit (equation (34)) as a function of the parameter $\omega$ for (a) $s=1$ and (b) $s=-1$. We use units such as $M=1, n=1, a=1$, and an upper bound $\varepsilon=10^{-7}$.

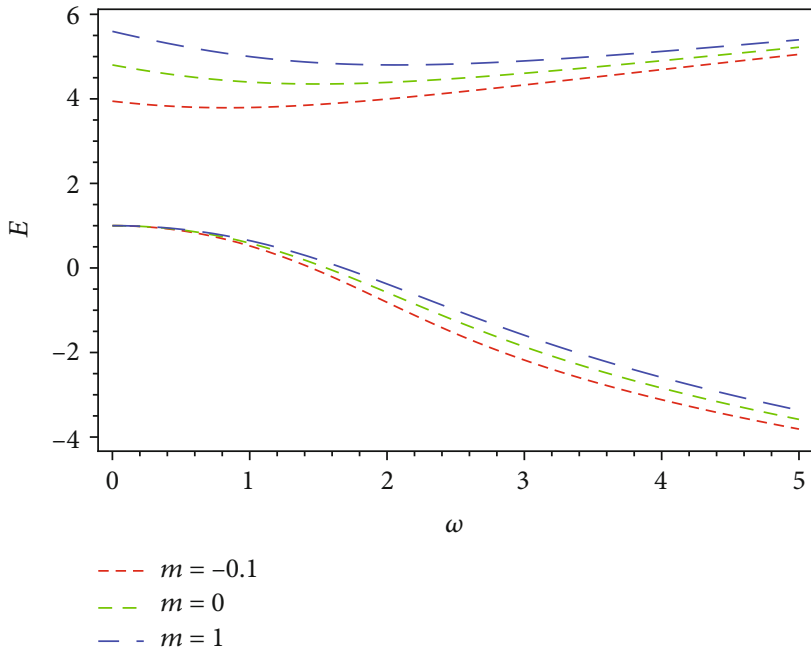

(a)

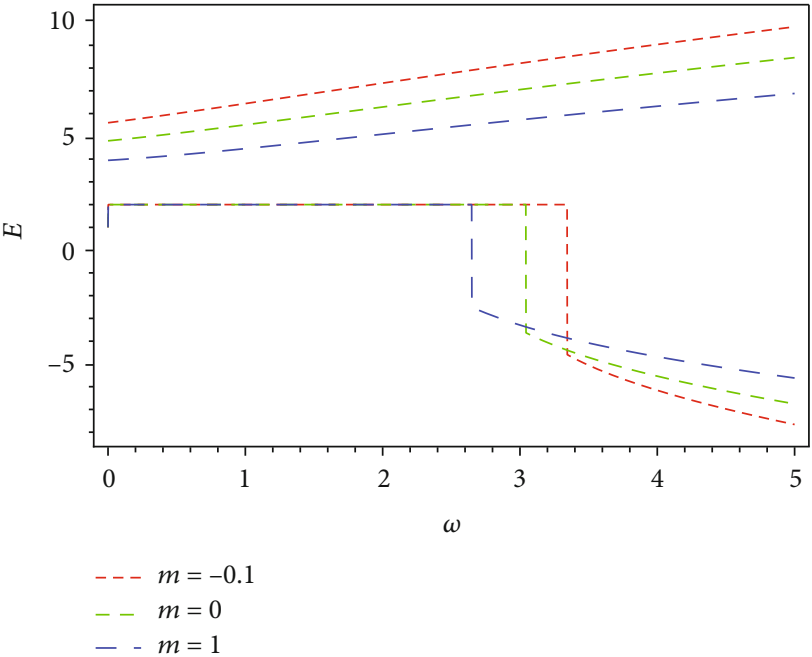

(b)

Figure 2: Illustration of the energy eigenvalues in the pseudospin symmetry limit (Eq. (35)) as a function of the parameter $\omega$ for (a) $s=1$ and (b) $s=-1$. We use units such as $M=1, n=1, a=1$ and an upper bound $\varepsilon=10^{-7}$.

the energy eigenvalues of Ref. [48] (after removing the magnetic flux parameter), which shows the consistency of the model in question. In particular, when $a$ and $\varepsilon$ are null, we obtain

$$
\begin{aligned}
& E^{2}-M^{2}=2 \omega[2 n+1+|m|-m-s], \\
& E^{2}-M^{2}=2 \omega[2 n+1+|m+s|-(m+s)-s],
\end{aligned}
$$

which are the usual relativistic Landau levels with the inclusion of the element of spin.
3.2. Particle Interacting with a Linear Potential. Let us consider the case where the particle interacts with a linear potential, br. As discussed above, linear interactions are included in the relativistic motion equations through the quadripotential $A_{\mu}$. In the case of the Dirac equation, depending on the configuration of external potentials involved, it can be solved exactly. An example of an exactly soluble model described by a linear interaction in relativistic quantum mechanics is the Dirac oscillator [60]. It is known that the Hamiltonian of the Dirac oscillator describes the interaction of a neutral particle with an electric field via an anomalous magnetic coupling [61]. In the 
context of confinement of quarks in mesons and baryons, it is well established that electrostatic linear potentials lead to the occurrence of the Klein paradox and particles cannot be confined [62]. To establish such confinement, we must invoke the scalar substitution (17). In this case, we make $V(r)= \pm S(r)=b r$ (where $b$ is a constant) in equation (26) to the limits of spin and pseudospin symmetries and proceed as before. The resulting equation is given by

$$
\begin{aligned}
{\left[\frac{d^{2}}{d r^{2}}\right.} & \left.+\left(\frac{1}{r}+\frac{\varepsilon b}{2}\right) \frac{d}{d r}-\frac{\left(m^{ \pm}\right)^{2}}{r^{2}}-\omega^{2} r^{2}-\mu^{ \pm} r\right] f_{ \pm}(r) \\
& -\left(\frac{k^{ \pm}}{r}-l^{ \pm}\right) f_{ \pm}(r)=0,
\end{aligned}
$$

with $m^{+}=m, m^{-}=m+s, \mu^{+}=2(E+M) b+3 \varepsilon \omega b s / 2, \mu^{-}=$ $2(E-M) b-3 \varepsilon \omega b s / 2, \quad k^{+}=\varepsilon b(1+3 s m) / 2, \quad k^{-}=\varepsilon b[1-3 s(m$ $+s)] / 2, \quad l^{+}=E^{2}-M^{2}+2 \omega(m+s)-2 M \varepsilon \omega s, \quad$ and $l^{-}=E^{2}-$ $M^{2}+2 \omega(m+s)+2 \omega s(1-\varepsilon M)$. In equation (37), the $+(-)$ signs refer to spin and pseudospin symmetries, respectively. By performing the variable change, $x=\sqrt{\omega} r$, equation (37) assumes the form

$$
\begin{gathered}
{\left[\frac{d^{2}}{d x^{2}}+\left(\frac{1}{x}+\frac{\kappa}{2}\right) \frac{d}{d x}-\frac{\left(m^{ \pm}\right)^{2}}{x^{2}}-x^{2}-a_{L}^{ \pm} x\right] f_{ \pm}(x)} \\
-\left(\frac{a_{C}^{ \pm}}{x}-\frac{l^{ \pm}}{\omega}\right) f_{ \pm}(x)=0,
\end{gathered}
$$

where we have defined the parameters $\kappa=\varepsilon b / \sqrt{\omega}, a_{L}^{ \pm}=\mu^{ \pm} / \omega$ $\sqrt{\omega}$, and $a_{C}^{ \pm}=k^{ \pm} / \sqrt{\omega}$. Note that the choice $V(r)=b r$ induces a Coulomb-like interaction in the resulting deformed sector of the eigenvalue equation. The origin of this Coulomb potential is due purely to the quantum deformation and boundary symmetries involved.

Equation (38) is of the Heun equation type, which is a homogeneous, linear, second-order, differential equation defined in the complex plane [63]. This equation can be put into its canonical form using the solution

$$
f_{ \pm}(x)=x^{\left|m^{ \pm}\right|} e^{-(1 / 2) x^{2}} e^{-(1 / 2)(a(+/ L)+(1 / 2) \kappa) x} y_{ \pm}(x),
$$

where $y_{ \pm}$satisfies the biconfluent Heun differential equation

$$
\begin{aligned}
y_{ \pm}^{\prime \prime} & +\left(\frac{\alpha^{ \pm}+1}{x}-2 x-\beta^{ \pm}\right) y_{ \pm}^{\prime}+\left(\gamma^{ \pm}-\alpha^{ \pm}-2\right) y_{ \pm} \\
& -\frac{1}{2 x}\left[\beta^{ \pm}\left(\alpha^{ \pm}+1\right)+\delta^{ \pm}\right] y_{ \pm}=0,
\end{aligned}
$$

with $\alpha^{ \pm}=2\left|m^{ \pm}\right|, \beta^{ \pm}=a_{L}^{ \pm}, \gamma^{ \pm}=\left(\beta^{ \pm}\right)^{2} / 4+l^{ \pm} / \omega$, and $\delta^{ \pm}=\kappa / 2$ $+2 a_{C}^{ \pm}$. Equation (40) has a regular singularity at $x=0$ and an irregular singularity at $\infty$ of rank 2 . Usually, the solution of this equation is given in terms of two linearly independent solutions as

$$
y_{ \pm}(x)=N\left(\alpha^{ \pm}, \beta^{ \pm}, \gamma^{ \pm}, \delta^{ \pm} ; x\right)+x^{-\alpha^{ \pm}} N\left(-\alpha^{ \pm}, \beta^{ \pm}, \gamma^{ \pm}, \delta^{ \pm} ; x\right),
$$

where (assuming that $\alpha^{ \pm}$is not a negative integer)

$$
N\left(\alpha^{ \pm}, \beta^{ \pm}, \gamma^{ \pm}, \delta^{ \pm} ; x\right)=\sum_{q=0}^{\infty} \frac{\mathscr{A}_{q}^{ \pm}\left(\alpha^{ \pm}, \beta^{ \pm}, \gamma^{ \pm}, \delta^{ \pm}\right)}{\left(1+\alpha^{ \pm}\right)_{q}} \frac{x^{q}}{q !}
$$

are the Heun functions. After the insertion of this solution into equation (40), we find $(q \geq 0)$

$$
\begin{gathered}
\mathscr{A}_{0}=1, \\
\mathscr{A}_{1}^{ \pm}=\frac{1}{2}\left[\delta^{ \pm}+\beta^{ \pm}\left(1+\alpha^{ \pm}\right)\right], \\
\mathscr{A}_{q^{ \pm}+2}^{ \pm}=\left\{(q+1) \beta^{ \pm}+\frac{1}{2}\left[\delta^{ \pm}+\beta^{ \pm}\left(1+\alpha^{ \pm}\right)\right]\right\} \mathscr{A}_{q+1}^{ \pm} \\
-(q+1)\left(q+1+\alpha^{ \pm}\right)\left[\gamma^{ \pm}-\alpha^{ \pm}-2-2 q\right] \mathscr{A}_{q}^{ \pm},
\end{gathered}
$$

where

$$
\left(1+\alpha^{ \pm}\right)_{q}=\frac{\Gamma\left(q+\alpha^{ \pm}+1\right)}{\Gamma\left(\alpha^{ \pm}+1\right)}, \quad q=0,1,2,3, \cdots .
$$

From the recursion relation (45), the function $N($ $\left.\alpha^{ \pm}, \beta^{ \pm}, \gamma^{ \pm}, \delta^{ \pm} ; x\right)$ becomes a polynomial of degree $n$ if and only if the two following conditions are imposed [63]:

$$
\begin{gathered}
\gamma^{ \pm}-\alpha^{ \pm}-2=2 n, \quad n=0,1,2, \cdots, \\
\mathscr{A}_{n+1}^{ \pm}=0,
\end{gathered}
$$

where $n$ is a positive integer. In this case, the $(n+1)$ th coefficient in the series expansion is a polynomial of degree $n$ in $\delta^{ \pm}$. When $\delta^{ \pm}$is a root of this polynomial, the $(n+1)$ th and subsequent coefficients cancel and the series truncates, resulting in a polynomial form of degree $n$ for $N\left(\alpha^{ \pm}, \beta^{ \pm}, \gamma^{ \pm}, \delta^{ \pm} ; x\right)$. From condition (47), we extract the energies at the spin and pseudosymmetry limits, given, respectively, by

$$
\begin{aligned}
E_{n m}^{2}-M^{2}= & 2 \omega(n+|m|+1)-\frac{b^{2}}{\omega^{2}}\left(E_{n m}+M\right)^{2} \\
& -\frac{3 b^{2}}{2 \omega}\left(E_{n m}+M\right) \varepsilon s+2 \omega[\varepsilon M s-(m+s)], \\
E_{n m}^{2}-M^{2}= & 2 \omega(n+|m+s|+1)-\frac{b^{2}}{\omega^{2}}\left(E_{n m}-M\right)^{2} \\
& +\frac{3 b^{2}}{2 \omega}\left(E_{n m}-M\right) \varepsilon s-2 \omega[s(1-\varepsilon M)+(m+s)] .
\end{aligned}
$$

The energy of a physical system must be a function involving all the parameters present in the equation of motion. In equation (49), the parameter $a_{C}^{ \pm}$is absent. However, it can be restored using condition (48) from which we 
obtain a relation between such parameter and the frequency of the system. For each value of $n$ fixed, we have a self-energy and its corresponding wave function. Let us consider solution (42) up to second order in $x$ of the expansion,

$N\left(\alpha^{ \pm}, \beta^{ \pm}, \gamma^{ \pm}, \delta^{ \pm} ; x\right)=\frac{\mathscr{A}_{0}}{\left(1+\alpha^{ \pm}\right)_{0}}+\frac{\mathscr{A}_{1}^{ \pm}}{\left(1+\alpha^{ \pm}\right)_{1}} x+\frac{\mathscr{A}_{2}^{ \pm}}{\left(1+\alpha^{ \pm}\right)_{2}} \frac{x^{2}}{2 !}+\cdots$

If we truncate the series in a term of order $x^{n}$, the resulting finite series is related, via solution (39), to the energy level $E_{n}$. Thus, the physical quantity that we can associate most closely with the series (50) truncated in the term $x^{n}$ is the energy $E_{n}$ of the particle with the wave function (39). In fact, it is necessary that the series (50) becomes an $n$-degree polynomial for the system to admit bound states. Thus, by using relation (45) and equations (43) and (44), the coefficient above $\mathscr{A}_{2}^{ \pm}$can be determined. If we want to truncate solution (50) in $x$, we must impose that $A_{1}^{ \pm}=0$ through condition (48); when we truncate in $x^{2}$, we make $A_{2}^{ \pm}=0$, and so on. For each of these cases, we can establish appropriate constraints between conditions (47) and (48). Since equation (49) is of the relativistic Landau level type, we prefer to fix the frequency $\omega$ in order to obtain an expression for the energies corresponding to each value of $n[41,64-70]$. Since it is done for both spin and pseudospin symmetries, we also label the frequency $\omega$ as $\omega_{n}^{ \pm}$, with the subscript $n$ characterizing the values to be fixed. After obtaining $\omega_{n}^{ \pm}$and replacing them in equation (49), we will have expressions for the energies involving the quantities $\alpha^{ \pm}, \beta^{ \pm}, \gamma^{ \pm}$, and $\delta^{ \pm}$, which contains the coupling constant $a_{C}^{ \pm}$, the mass of the particle $M$, and the quantum angular momentum number $m^{ \pm}$. Thus, for $A_{1}^{ \pm}=0$, it means that we are investigating the particular solution for $n=0$. In this case, from equation (44), we have

$$
\frac{1}{2}\left(\delta^{ \pm}+\beta^{ \pm} \tilde{m}^{ \pm}\right)=0
$$

where $\tilde{m}^{ \pm}=1+\alpha^{ \pm}$. Solving (51) for $\omega_{0}^{ \pm}$, we get the relations

$$
\begin{aligned}
& \omega_{0}^{+}=-\frac{2\left(E_{0 m}^{+}+M\right)(1+2|m|)}{\varepsilon[3 s(|m|+m)+3 / 2(s+1)]}, \\
& \omega_{0}^{-}=\frac{2\left(E_{0 m}^{-}-M\right)(1+2|m+s|)}{\varepsilon[3 s(|m+s|+m+s)+(3 / 2)(s-1)]} .
\end{aligned}
$$

Proceeding in a similar way to $\mathscr{A}_{2}^{ \pm}=0$, we find the following third-degree polynomial in $\omega_{1}^{ \pm}(n=1)$ :

$$
\mathscr{A}^{ \pm}\left[\omega_{1}^{ \pm}\right]^{3}+\mathscr{B}^{ \pm}\left[\omega_{1}^{ \pm}\right]^{2}+\mathscr{C}^{ \pm}\left[\omega_{1}^{ \pm}\right]+\mathscr{D}^{ \pm}=0,
$$

with

$$
\begin{aligned}
& \mathscr{A}^{+}=2(1+2|m|), \\
& \mathscr{A}^{-}=2(1+2|m+s|), \\
& \mathscr{B}^{+}=\mathscr{B}^{-}=0, \\
& \mathscr{C}^{+}=-\frac{3}{2} \varepsilon b^{2}\left(E_{1 m}^{+}+M\right)\left[(2|m|+1) s+C^{+}\right], \\
& \mathscr{C}^{-}=\frac{3}{2} \varepsilon b^{2}\left(E_{1 m}^{-}-M\right)\left[s(2|m+s|+1)+C^{-}\right], \\
& \mathscr{D}^{+}=-b^{2}\left(E_{1 m}^{+}+M\right)^{2}(1+2|m|)(3+2|m|), \\
& \mathscr{D}^{-}=-b^{2}\left(E_{1 m}^{-}-M\right)^{2}(1+2|m+s|)(3+2|m+s|),
\end{aligned}
$$

where $C^{+}=2(1+|m|)(2 s|m|+2 m s+s+1)$ and $C^{-}=2(\mid m+$ $s \mid+1)(2 s(|m+s|+m)+s+1)$. Equation (53) has only one real root. For each specific frequency, $\omega_{0}^{ \pm}$and $\omega_{1}^{ \pm}$, we can determine the energies $E_{0 m}^{ \pm}$and $E_{1 m}^{ \pm}$by the following expressions:

$$
\begin{aligned}
\left(E_{0 m}^{+}\right)^{2}-M^{2}= & 2 \omega_{0}^{+}(|m|+1)-\frac{b^{2}}{\left(\omega_{0}^{+}\right)^{2}}\left(E_{0 m}^{+}+M\right)^{2} \\
& -\frac{3 b^{2}}{2 \omega_{0 m}^{+}}\left(E_{0}^{+}+M\right) \varepsilon s+2 \omega_{0}^{+}[\varepsilon M s-(m+s)],
\end{aligned}
$$

$$
\begin{aligned}
\left(E_{0 m}^{-}\right)^{2}-M^{2}= & 2 \omega_{0}^{-}(|m+s|+1)-\frac{b^{2}}{\left(\omega_{0}^{-}\right)^{2}}\left(E_{0 m}^{-}-M\right)^{2} \\
& +\frac{3 b^{2}}{2 \omega_{0}^{-}}\left(E_{0 m}^{-}-M\right) \varepsilon s \\
& -2 \omega_{0}^{-}[s(1-\varepsilon M)+(m+s)], \\
\left(E_{1 m}^{+}\right)^{2}-M^{2}= & 2 \omega_{1}^{+}(|m|+2)-\frac{b^{2}}{\left(\omega_{1}^{+}\right)^{2}}\left(E_{1 m}^{+}+M\right)^{2} \\
& -\frac{3 b^{2}}{2 \omega_{1}^{+}}\left(E_{1 m}^{+}+M\right) \varepsilon s+2 \omega_{1}^{+}[\varepsilon M s-(m+s)],
\end{aligned}
$$

$$
\begin{aligned}
\left(E_{1 m}^{-}\right)^{2}-M^{2}= & 2 \omega_{1}^{-}(|m+s|+2)-\frac{b^{2}}{\left(\omega_{1}^{-}\right)^{2}}\left(E_{1 m}^{-}-M\right)^{2} \\
& +\frac{3 b^{2}}{2 \omega_{1}^{-}}\left(E_{1 m}^{-}-M\right) \varepsilon s \\
& -2 \omega_{1}^{-}[s(1-\varepsilon M)+(m+s)],
\end{aligned}
$$

where $\omega_{1}^{ \pm}$is given by

$$
\omega_{1}^{ \pm}=\sqrt[3]{\frac{\Delta^{ \pm}}{18}} \frac{1}{\mathscr{A}^{ \pm}}-\sqrt[3]{\frac{2}{3 \Delta^{ \pm}}} \mathscr{C}^{ \pm},
$$

with

$$
\Delta^{ \pm}=\sqrt{3} \sqrt{27\left[\mathscr{A}^{ \pm}\right]^{4}\left[\mathscr{D}^{ \pm}\right]^{2}+4\left[\mathscr{A}^{ \pm}\right]^{3}\left[\mathscr{C}^{ \pm}\right]^{3}}-9\left[\mathscr{A}^{ \pm}\right]^{2} \mathscr{D}^{ \pm},
$$




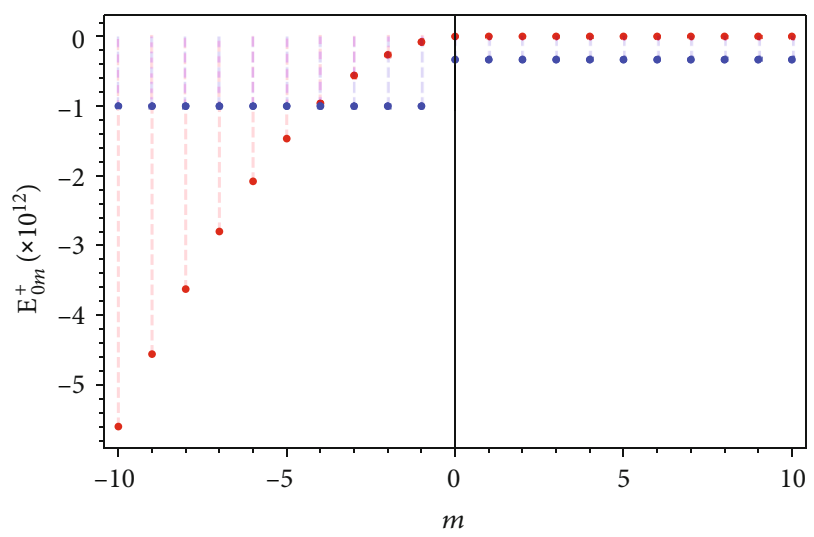

(a)

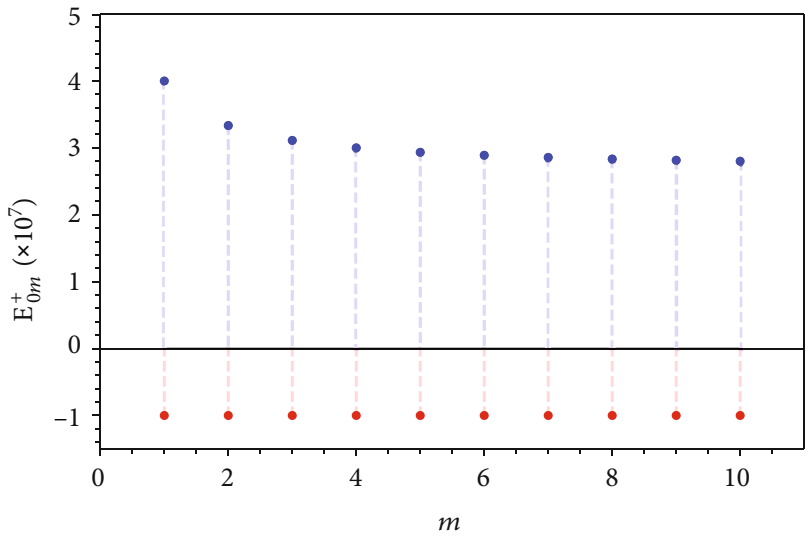

(b)

FIGURE 3: Illustration of the energy eigenvalues in the spin symmetry limit (equation (55)) as a function of the parameter $m$ for (a) $s=1$ and (b) $s=-1$. We use units such as $M=1, n=1, b=1$, and $\varepsilon=10^{-7}$.

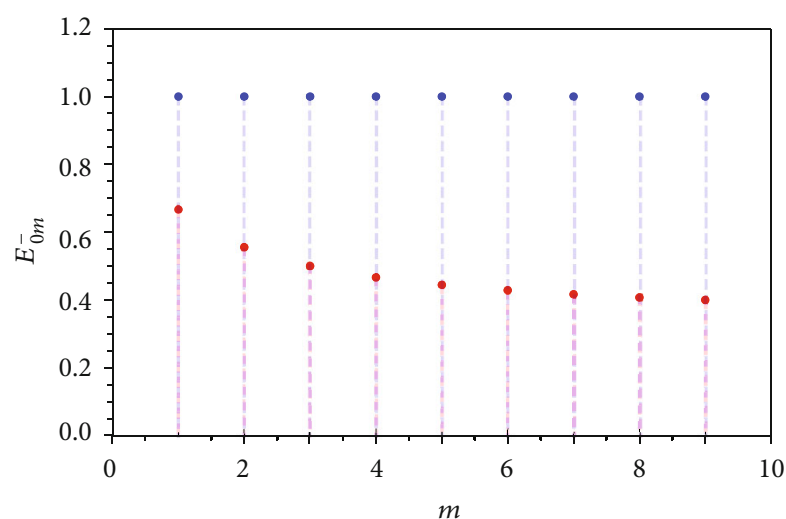

(a)

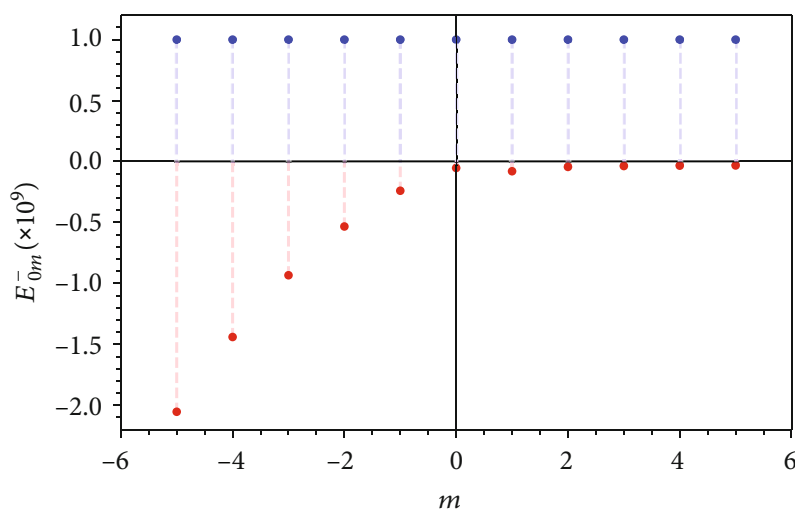

(b)

FIGURE 4: Illustration of the energy eigenvalues in the pseudospin symmetry limit (equation (56)) as a function of the parameter $m$ for (a) $s=1$ and (b) $s=-1$. We use units such as $M=1, n=1, b=1$, and $\varepsilon=10^{-7}$.

and the requirement that $27\left[\mathscr{A}^{ \pm}\right]^{4}\left[\mathscr{D}^{ \pm}\right]^{2}+4\left[\mathscr{A}^{ \pm}\right]^{3}\left[\mathscr{C}^{ \pm}\right]^{3}>0$. Such energies represent the first two energy levels of the system. For simplicity, we perform a numerical analysis only for the case $n=0$. In this way, by studying equation (55), we find that for $s=1$ and $m>0$, the energy of the particle is infinitely degenerate, with respective eigenvalues $E_{0 m}^{+}=-1$ and $E_{0 m}^{+}=-0.33$, whereas for $m<0$, only one of the roots is infinitely degenerate with energy $E_{0 m}^{+}=-1$ (Figure 3(a)). On the other hand, when we analyze equation (55) for $s=-1$, we verify that the energy spectrum is defined only for $m>0$, and one of the roots is infinitely degenerate with eigenvalue $E_{0 m}^{+}=-1$ (Figure $3(\mathrm{~b})$ ). These characteristics are also present in the energies from equation (56). For $s=1$, the energies are defined only for $m>0$ and one of the roots is infinitely degenerate, with eigenvalue $E_{0 m}^{-}=1$ (Figure 4(a)). For $s=-1$ and $m<0$, there is an infinitely degenerate root with eigenvalue $E_{0 m}^{-}=1$ while for $m>$ 0 both roots are infinitely degenerate with respective energies $E_{0 m}^{-}=1$ and $E_{0 m}^{-}=-2.7 \times 10^{7}$ (Figure $4(\mathrm{~b})$ ).

To determine the energies corresponding to $n=2,3,4, \cdots$, we must make use of the above recipe. However, as we can see from equation (53), the polynomials of degree $n \geq 3$ resulting from condition (48), in general, not all roots are physically acceptable. In the absence of deformation, the resulting equation describes the motion of an electron subject to interactions (15), (16), and (17). In the literature, models in this context can be found. With the presence of these interactions in the Dirac equation, the wave function depends on several parameters. Thus, if we consider a null deformation, a direct comparison with particular cases in the literature is not immediate. The reader can see some examples in Refs. [41, 71-73].

\section{Conclusions}

We have studied the relativistic quantum dynamics of a spin $1 / 2$ charged particle with minimal and scalar couplings in the quantum deformed framework generated by the $\kappa$-Poincaré-Hopf algebra. The problem has been formulated using the $\kappa$-deformed Dirac equation in two dimensions. The $\kappa$-deformed Pauli equation was derived to study the dynamics of the system taking into account 
the spin and pseudospin symmetry limits. For the $\kappa$ -deformed Dirac-Pauli equation obtained (equation (26)), we have argued that only particular choices of radial function $V(r)$ lead to exactly solvable differential equations. We have considered the case where the particle interacts with a uniform magnetic field, a planar harmonic oscillator, and a linear potential. We have verified that the linear potential leads to a Coulomb-type term in the $\kappa$-deformed sector of the radial equation. The resulting equation obtained is a Heun-type differential equation. Analytical solutions for both spin and pseudospin symmetry limits enabled us to obtain expressions for the energy eigenvalues (through the use of equations (47) and (48)) and wave functions. Because of the limitations imposed by condition (48), we have derived expressions for the energies corresponding only to $n=0$ (equations (55) and (56)) and $n=1$ (equations (57) and (58)). We have shown that the energy eigenvalues and wave functions are modified by both the spin element $s$ and the deformation parameter $\varepsilon$. We believe that future experiments may provide some estimate on the magnitude of the deformation parameter within the context of the model studied.

\section{Data Availability}

We did not use any data in this paper.

\section{Conflicts of Interest}

The authors declare that there is no conflict of interests regarding the publication of this paper.

\section{Acknowledgments}

EOS acknowledges funding from Conselho Nacional de Desenvolvimento Científico e Tecnológico (CNPq) (Grant Nos. 427214/2016-5 and 303774/2016-9) and Fundação de Amparo à Pesquisa e ao Desenvolvimento Científico e Tecnológico do Maranhão (FAPEMA) (Grant Nos. 01852/14 and 01202/16).

\section{References}

[1] J. Lukierski, A. Nowicki, and H. Ruegg, "New quantum Poincaré algebra and $\kappa$-deformed field theory," Physics Letters $B$, vol. 293, no. 3-4, pp. 344-352, 1992.

[2] J. Lukierski, H. Ruegg, A. Nowicki, and V. N. Tolstoy, "qdeformation of Poincaré algebra," Physics Letters B, vol. 264, no. 3-4, pp. 331-338, 1991.

[3] A. Nowicki, E. Sorace, and M. Tarlini, "The quantum deformed Dirac equation from the algebra," Physics Letters B, vol. 302, no. 4, pp. 419-422, 1993.

[4] L. C. Biedenharn, B. Mueller, and M. Tarlini, "The DiracCoulomb problem for the $\kappa$-Poincare quantum group," Physics Letters B, vol. 318, no. 4, pp. 613-616, 1993.

[5] J. Lukierski and H. Ruegg, "Quantum $\kappa$-Poincaré in any dimension,” Physics Letters B, vol. 329, no. 2-3, pp. 189-194, 1994.

[6] S. Majid and H. Ruegg, "Bicrossproduct structure of $\kappa$-Poincare group and non-commutative geometry," Physics Letters $B$, vol. 334, no. 3-4, pp. 348-354, 1994.
[7] J. Lukierski, H. Ruegg, and W. J. Zakrzewski, "Classical and quantum mechanics of free $\kappa$-relativistic systems," Annals of Physics, vol. 243, no. 1, pp. 90-116, 1995.

[8] M. Arzano, J. Kowalski-Glikman, and A. Walkus, "Lorentz invariant field theory on $\kappa$-Minkowski space," Classical and Quantum Gravity, vol. 27, no. 2, article 025012, 2010.

[9] D. Kovačević, S. Meljanac, A. Pachoł, and R. Štrajn, "Generalized Poincare algebras, Hopf algebras and $\kappa$-Minkowski spacetime," Physics Letters B, vol. 711, no. 1, pp. 122-127, 2012.

[10] P. Kosiński, J. Lukierski, and P. Maślanka, “ $\kappa$-Deformed Wigner construction of relativistic wave functions and free fields on $\kappa$-Minkowski space," Nuclear Physics B - Proceedings Supplements, vol. 102-103, pp. 161-168, 2001.

[11] M. V. Cougo-Pinto, C. Farina, and J. F. M. Mendes, "Casimir effect and creation of radiation in confined $\kappa$-deformed electrodynamics," Physics Letters B, vol. 529, no. 3-4, pp. 256260, 2002.

[12] E. Harikumar, T. Jurić, and S. Meljanac, "Electrodynamics on -Minkowski space-time," Physical Review D, vol. 84, no. 8, article 085020, 2011.

[13] M. Dimitrijević and L. Jonke, “A twisted look on kappa-Minkowski: U(1) gauge theory," Journal of High Energy Physics, vol. 1112, p. 80, 2011.

[14] T. Jurić, S. Meljanac, and R. Štrajn, "Differential forms and $\kappa$ Minkowski spacetime from extended twist," European Physical Journal C: Particles and Fields, vol. 73, no. 7, p. 2472, 2013.

[15] A. Borowiec and A. Pachol, " $\kappa$-Minkowski spacetime as the result of Jordanian twist deformation," Physical Review D, vol. 79, no. 4, article 045012, 2009.

[16] S. Meljanac and M. Stojić, "New realizations of Lie algebra kappa-deformed Euclidean space," European Physical Journal C: Particles and Fields, vol. 47, no. 2, p. 531, 2006.

[17] S. Meljanac, A. Samsarov, M. Stojić, and K. S. Gupta, " $\kappa$-Minkowski spacetime and the star product realizations," European Physical Journal C: Particles and Fields, vol. 53, no. 2, pp. 295309, 2008.

[18] F. M. Andrade and E. O. Silva, "Effects of quantum deformation on the spin-1/2 Aharonov-Bohm problem," Physics Letters $B$, vol. 719, no. 4-5, pp. 467-471, 2013.

[19] A. Agostini, G. Amelino-Camelia, and M. Arzano, "Dirac spinors for doubly special relativity and $\kappa$-Minkowski noncommutative spacetime," Classical and Quantum Gravity, vol. 21, no. 8, pp. 2179-2202, 2004.

[20] R. Aloisio, A. Galante, A. F. Grillo, F. Mendez, J. M. Carmona, and J. L. Cortes, "Particle and antiparticle sectors in DSR1 and $\kappa$-Minkowski space-time," Journal of High Energy Physics, vol. 2004, no. 5, p. 28, 2004.

[21] P. Roy and R. Roychoudhyry, "Aharonov-Bohm interaction for a deformed non-relativistic spin 12 particle," Physics Letters B, vol. 359, no. 3-4, pp. 339-342, 1995.

[22] P. Roy and R. Roychoudhury, "A note on the $\kappa$ deformed Landau problem," Physics Letters B, vol. 339, no. 1-2, pp. 87-89, 1994.

[23] S. Meljanac, A. Pachoł, A. Samsarov, and K. S. Gupta, "Different realizations of $\kappa$-momentum space," Physical Review D, vol. 87, no. 12, article 125009, 2013.

[24] K. S. Gupta, S. Meljanac, and A. Samsarov, "Quantum statistics and noncommutative black holes," Physical Review D, vol. 85, no. 4, article 045029, 2012. 
[25] T. R. Govindarajan, K. S. Gupta, E. Harikumar, S. Meljanac, and D. Meljanac, "Deformed oscillator algebras and QFT in א-Minkowski spacetime," Physical Review D, vol. 80, no. 2, article 025014, 2009.

[26] M. Dimitrijević, L. Jonke, L. Moller, E. Tsouchnika, J. Wess, and M. Wohlgenannt, "Deformed field theory on $\kappa$-spacetime," European Physical Journal C: Particles and Fields, vol. 31, no. 1, pp. 129-138, 2003.

[27] F. M. Andrade, E. O. Silva, M. M. Ferreira Jr., and E. C. Rodrigues, "On the $\kappa$-Dirac oscillator revisited," Physics Letters $B$, vol. 731, pp. 327-330, 2014.

[28] F. M. Andrade and E. O. Silva, "The 2D $\kappa$-Dirac oscillator," Physics Letters B, vol. 738, pp. 44-47, 2014.

[29] F. M. Andrade, E. O. Silva, D. Assafrão, and C. Filgueiras, "Effects of quantum deformation on the integer quantum Hall effect," EPL (Europhysics Letters), vol. 116, no. 3, 2016.

[30] G. B. Smith and L. J. Tassie, "Excited states of mesons and the quark-antiquark interaction," Annals of Physics, vol. 65, no. 1, pp. 352-360, 1971.

[31] J. S. Bell and H. Ruegg, "Dirac equations with an exact higher symmetry," Nuclear Physics B, vol. 98, no. 1, pp. 151-153, 1975.

[32] J. N. Ginocchio, "Pseudospin as a relativistic symmetry," Physical Review Letters, vol. 78, no. 3, pp. 436-439, 1997.

[33] J. N. Ginocchio, "Relativistic symmetries in nuclei and hadrons," Physics Reports, vol. 414, no. 4-5, pp. 165-261, 2005.

[34] K. Sugawara-Tanabe, J. Meng, S. Yamaji, and A. Arima, “The pseudo-spin symmetry in a Dirac equation," Journal of Physics G: Nuclear and Particle Physics, vol. 25, no. 4, pp. 811-813, 1999.

[35] P. Alberto, M. Malheiro, T. Frederico, and A. de Castro, "General spin and pseudospin symmetries of the Dirac equation," Physical Review A, vol. 92, no. 6, article 062137, 2015.

[36] H. Hassanabadi, E. Maghsoodi, and S. Zarrinkamar, "Spin and pseudospin symmetries of Dirac equation and the Yukawa potential as the tensor interaction," Communications in Theoretical Physics, vol. 58, no. 6, pp. 807-814, 2012.

[37] H. Akcay and R. Sever, "Approximate analytical solutions of Dirac equation with spin and pseudo spin symmetries for the diatomic molecular potentials plus a tensor term with any angular momentum," Few-Body Systems, vol. 54, no. 11, pp. 1839-1850, 2013.

[38] O. Aydoğdu and R. Sever, "Pseudospin and spin symmetry in the Dirac equation with Woods-Saxon potential and tensor potential," The European Physical Journal A, vol. 43, no. 1, p. 73, 2009.

[39] S. M. Ikhdair and R. Sever, "Solutions of the spatiallydependent mass Dirac equation with the spin and pseudospin symmetry for the Coulomb-like potential," Applied Mathematics and Computation, vol. 216, no. 2, pp. 545-555, 2010.

[40] A. S. de Castro and P. Alberto, "Spin and pseudospin symmetries in the Dirac equation with central Coulomb potentials," Physical Review A, vol. 86, no. 3, article 032122, 2012.

[41] L. B. Castro, "Relating pseudospin and spin symmetries through chiral transformation with tensor interaction," Physical Review C, vol. 86, no. 5, article 052201, 2012.

[42] L. B. Castro, A. S. de Castro, and P. Alberto, "Pseudospin and spin symmetries in 1+1 dimensions: the case of the Coulomb potential," Annals of Physics, vol. 356, pp. 83-94, 2015.

[43] F. S. Azevedo, E. O. Silva, L. B. Castro, C. Filgueiras, and D. Cogollo, "Relativistic quantum dynamics of a neutral parti- cle in external electric fields: an approach on effects of spin," Annals of Physics, vol. 362, pp. 196-207, 2015.

[44] W. Greiner, Relativistic Quantum Mechanics. Wave Equations, Springer, 2000.

[45] R. H. Brandenberger, A.-C. Davis, and A. M. Matheson, "Callan-Rubakov effect for strings," Nuclear Physics B, vol. 307, no. 4, pp. 909-923, 1988.

[46] C. R. Hagen and D. K. Park, "Relativistic Aharonov-BohmCoulomb problem," Annals of Physics, vol. 251, no. 1, pp. 45-63, 1996.

[47] F. M. Andrade and E. O. Silva, "Effects of spin on the dynamics of the 2D Dirac oscillator in the magnetic cosmic string background," European Physical Journal C: Particles and Fields, vol. 74, no. 12, article 3187, 2014.

[48] L. B. Castro and E. O. Silva, "Quantum dynamics of a spin-1/2 charged particle in the presence of a magnetic field with scalar and vector couplings," The European Physical Journal C, vol. 75 , no. 7, p. 321, 2015.

[49] L. D. Landau and E. M. Lifschitz, Quantum Mechanics, Pergamon, Oxford, 1981.

[50] L. Kantorovich, Quantum Theory of the Solid State: An Introduction, Fundamental Theories of Physics, Springer Netherlands, 2004.

[51] A. I. Landau, A. S. Kovalev, and A. D. Kondratyuk, "Model of interacting atomic chains and its application to the description of the crowdion in an anisotropic crystal," Physica Status Solidi (B), vol. 179, no. 2, pp. 373-381, 1993.

[52] D. Bacon, Y. Osetsky, and D. Rodney, "Chapter 88 dislocationobstacle interactions at the atomic level," Dislocations in Solids, vol. 15, pp. 1-90, 2009.

[53] C. Wang, W. Duan, L. Glazman, and A. Alexandradinata, "Landau quantization of nearly degenerate bands and full symmetry classification of Landau level crossings," Physical Review B, vol. 100, no. 1, article 014442, 2019.

[54] L.-J. Yin, K.-K. Bai, W.-X. Wang, S.-Y. Li, Y. Zhang, and L. He, "Landau quantization of Dirac fermions in graphene and its multilayers," Frontiers of Physics, vol. 12, no. 4, article 127208, 2017.

[55] C. Furtado, A. Rosas, and S. Azevedo, "Landau quantization and curvature effects in a two-dimensional quantum dot," Europhysics Letters (EPL), vol. 79, no. 5, p. 57001, 2007.

[56] B. F. Ramos, I. A. Pedrosa, and K. Bakke, "Effects of a nonHermitian potential on the Landau quantization," International Journal of Modern Physics A, vol. 34, no. 12, article 1950072, 2019.

[57] Q. Haque and S. A. Shan, "Influence of Landau quantization and temperature degeneracy on drift acoustic waves in electron-positron-ion plasmas," Physics of Plasmas, vol. 26, no. 1, article 012111, 2019.

[58] L. Deych, Advanced Undergraduate Quantum Mechanics: Methods and Applications, Springer International Publishing, 2018.

[59] F. W. J. Olver, D. W. Lozier, R. F. Boisvert, and C. W. Clark, Eds., NIST Handbook of Mathematical Functions, Cambridge University Press, 2010.

[60] M. Moshinsky and A. Szczepaniak, "The Dirac oscillator," Journal of Physics A, vol. 22, no. 17, pp. L817-L819, 1989.

[61] R. P. Martinez-y Romero, H. N. Nunez-Yepez, and A. L. Salas-Brito, "Relativistic quantum mechanics of a Dirac oscillator," European Journal of Physics, vol. 16, no. 3, pp. 135-141, 1995. 
[62] F. Domínguez-Adame and M. A. González, "Solvable linear potentials in the Dirac equation," Europhysics Letters, vol. 13, no. 3, pp. 193-198, 1990.

[63] P. Ronveaux, A. Ronveaux, F. Arscott et al., Heun's Differential Equations, Oxford Sscience Ppublications, Oxford University Press, 1995.

[64] A. B. Oliveira and K. Bakke, "On the effects on a Landau-type system for an atom with no permanent electric dipole moment due to a Coulomb-type potential," Annals of Physics, vol. 365, pp. 66-72, 2016.

[65] E. R. Arriola, A. Zarzo, and J. S. Dehesa, "Spectral properties of the biconfluent Heun differential equation," Journal of Computational and Applied Mathematics, vol. 37, no. 1-3, pp. 161-169, 1991.

[66] F. Caruso, J. Martins, and V. Oguri, "Solving a two-electron quantum dot model in terms of polynomial solutions of a Biconfluent Heun equation," Annals of Physics, vol. 347, pp. 130-140, 2014.

[67] R. L. L. Vitória, C. Furtado, and K. Bakke, "On a relativistic particle and a relativistic position-dependent mass particle subject to the Klein-Gordon oscillator and the Coulomb potential," Annals of Physics, vol. 370, pp. 128-136, 2016.

[68] K. Bakke and C. Furtado, "On the Klein-Gordon oscillator subject to a Coulomb-type potential," Annals of Physics, vol. 355, pp. 48-54, 2015.

[69] K. Bakke and H. Belich, "Rashba coupling induced by Lorentz symmetry breaking effects," Annalen der Physik, vol. 526, no. 3-4, pp. 187-194, 2014.

[70] D. F. Lima, F. M. Andrade, L. B. Castro, C. Filgueiras, and E. O. Silva, "On the 2D Dirac oscillator in the presence of vector and scalar potentials in the cosmic string spacetime in the context of spin and pseudospin symmetries," The European Physical Journal C, vol. 79, no. 7, p. 596, 2019.

[71] M. J. Bueno, C. Furtado, and K. Bakke, "On the effects of a screw dislocation and a linear potential on the harmonic oscillator," Physica B: Condensed Matter, vol. 496, pp. 45-48, 2016.

[72] A. B. Oliveira and K. Bakke, "On the Landau system for an atom with no permanent electric dipole moment subject to a linear confining potential," International Journal of Modern Physics A, vol. 31, no. 6, article 1650019, 2016.

[73] S. M. Ikhdair, "Exact Solution of Dirac Equation with Charged Harmonic Oscillator in Electric Field: Bound States," Journal of Modern Physics, vol. 3, no. 2, pp. 170-179, 2012. 